\title{
Factors affecting schedule-induced wood-chewing in rats: Percentage and rate of reinforcement, and operant requirement
}

\author{
T. J. ROPER, L. EDWARDS, and G. CROSBLAND \\ University of Sussex, Brighton, England
}

\begin{abstract}
In Experiment 1, rats were allowed to acquire either schedule-induced drinking or scheduleinduced wood-chewing behavior under a fixed-interval (FI) 60-6ec schectule of food reinforcement, following which food was omitted from $20 \%$ and then $50 \%$ of interreinforcement intervals. Omiosion of food severely diorupted induced drinking but had relatively little effect on induced wood-chewing. Experiment 2 investigated wood-chewing as a function of reinforcement rate, using a range of FI schedules from 5 to $180 \mathrm{wec}$ in duration. Both the amount of chewing per session and the relative time epent chewing were bitonically related to reinforcement rate. In Experiment 3, schedule-induced chewing that had been acquired under a reaponse-dependent schedule was found to persiat under a respones-independent schedule. Induced wood-chewing resembles other induced behaviors in important respects, but quantitative differences are also apparent.
\end{abstract}

Falk (1961) showed that when rats were allowed to earn food pellets intermittently, in the presence of a water source, they developed the habit of drinking a small amount of water within each interpellet interval. Because it dramatically enhances the rat's water intake, this type of drinking has become known as "schedule-induced polydipsia," or SIP. Two decades of research have revealed SIP to be an extraordinarily reliable effect, by behavioral standards, and have produced a wealth of experimental results and of conflicting theoretical accounts (see reviews by Falk, 1971, 1977, and Staddon, 1977). Nevertheless, no satisfactory explanation of the rat's enhanced drinking under intermittent schedules of food availability has yet emerged.

We have chosen to address the problem of SIP obliquely, by asking whether the phenomenon of schedule induction extends to activities other than drinking, to reinforcers other than food, and to species other than the rat. In this way, we hope to determine the extent to which SIP depends on special characteristics of the relationship between eating and drinking in the rat, as opposed to reflecting the operation of more general principles. We have recently confirmed that wood-chewing can occur as a schedule-induced behavior in rats when food is intermittently available, and have shown that induced wood-chewing (like SIP) is inversely related to level of food deprivation (Roper \& Crossland, 1982). This finding supports the idea that schedule induc-

We thank the SRC for financial support. Reprints may be obtained from: T. J. Roper, School of Biology, University of Sussex, Brighton BN1 9QG, England. tion is a general phenomenon (for a critical review of other relevant evidence, see Roper, 1981). On the other hand, schedule-induced wood-chewing has so far proved relatively variable in its rate and probability of acquisition, and it differs from SIP in the precise temporal location of the behavior within the interfood interval (Roper Crossland, 1981). Thus, induced activities other than drinking may not resemble SIP in all respects.

In this paper, we pursue the analogy between wood-chewing and SIP by examining the effect of omitting occasional food reinforcements, of varying the rate of food reinforcement, and of switching from a response-dependent to a response-independent schedule.

\section{EXPERIMENT 1}

Several studies of schedule-induced drinking have used second-order schedules of food reinforcement, in which one schedule component results in delivery of food while another results in presentation of a nonfood discriminative stimulus. All these studies show that drinking occurs less frequently, and that bouts of drinking are shorter, following a nonfood discriminative stimulus than following food. There is, however, disagreement about the precise extent to which SIP is disrupted (see review by CorfieldSumner, Blackman, \& Stainer, 1977).

Experiment 1 followed a study by Allen, Porter, and Arazie (1975), who allowed rats to develop SIP under a fixed-interval (FI) 60-sec schedule, and then caused food to be omitted from specified percentages of interreinforcement intervals. In our exper- 
iment, rats were allowed to acquire the behavior of schedule-induced wood-chewing, following which food was omitted from $20 \%$ and $50 \%$ of interreinforcement intervals. In addition, we subjected a few polydipsic rats to the same procedure, as a confirmation of Allen et al.'s results.

\section{Method}

\section{Subjects}

Twelve naive fernale hooded rats (mean weight $208 \mathrm{~g}$ ) were obtained from the laboratory colony and assigned at random to two groups: Group $C$ (for "chew"), $N=9$, and Group D (for "drink"), $\mathbf{N}=3$. The rats were housed individually under a reversed light cycle (light on from 2000 to $0800 \mathrm{~h}$ ), and were tested at $80 \%$ of free-feeding weight.

\section{Apparatus}

The apparatus consisted of four operant chambers, each of which contained a response lever and a food tray mounted $10 \mathrm{~cm}$ apart on the same wall of the chamber (for details, see Roper \& Crossland, 1982). Complete-diet 45-mg food pellets (Campden Instruments Ltd.) were delivered automatically. Three chambers (used for testing the Group $\mathrm{C}$ rats) also contained a "chewometer" mounted on the opposite wall from the lever and food tray. This consisted of a block of oak $(4 \times 3 \times 2 \mathrm{~cm})$ bolted onto the face of a pigeon key (Roper, 1978). The fourth chamber (used for testing the Group D rats) contained a drinking spout connected to a drinkometer circuit (see Roper \& Nieto, 1979). Each chamber was housed in a sound-attenuating chest fitted with a one-way observation window.

\section{Procedure}

Phase 1: Pretraining (15 rexsions). Following magazine training and shaping, leverpressing was reinforced according to an FI 60-sec schedule during 10 60-min sessions. Pellet delivery was signaled by 2.5 -sec illumination of a light above the food tray, as well as by the sound of the dispenser. During pretraining, no wood block or water spout was present, because experience had shown that prior adaptation to an FI $60-\mathbf{s e c}$ schedule facilitated the subsequent appearance of induced wood-chewing (Roper \& Crossland, 1982).

Phase 2: Acqulation of induced behavior (26 sessions). A wood block or water spout was introduced into each chamber, and the Fl 60-sec schedule was continued. All three of the Group D rats became polydipsic, but two of the Group $\mathrm{C}$ rats failed to show wood-chewing behavior and were dropped from the experiment.

Phase 3: 20\% omission (10 sesaions). The procedure was the same as in Phase 2, except that food was omitted at the end of every fifth fixed interval. The $2.5-\mathrm{sec}$ stimulus light was presented on completion of every interval.

Phase 4: 50\% omiseion (10 sessions). Food was omitted following the completion of alternate fixed intervals.

Wood-block displacements and water-spout contacts were recorded automatically, and direct observations were made periodically via closed-circuit video.

\section{Results}

\section{Phases 1 and 2: Acquisition of Induced Behavior}

Figure 1 shows the acquisition of schedule-induced wood-chewing during Phase 2 of the experiment, in seven of the nine rats in Group $C$. The other two rats showed little or no wood-chewing behavior. Figure 2 shows the acquisition of schedule-induced drinking in all three rats in Group D. By comparing

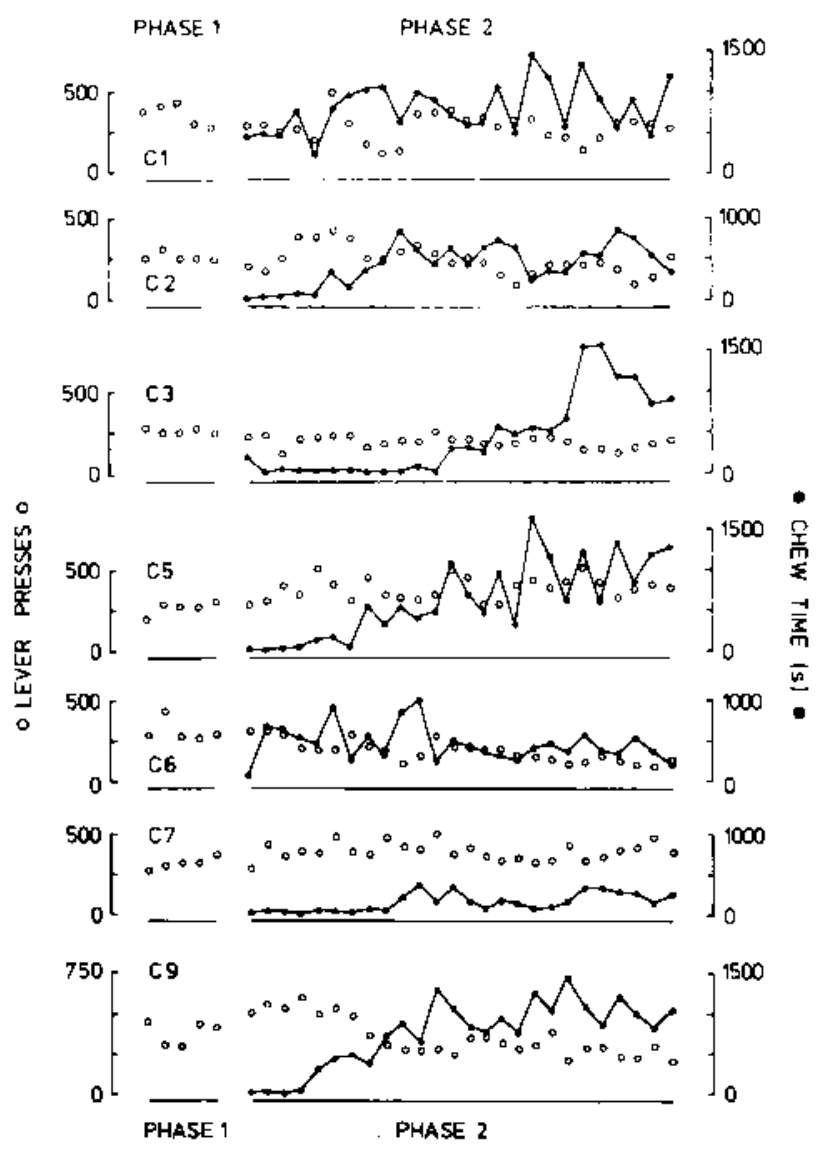

Figure 1. Number of leverpresses per sescion (open circles) and chew time per session (filled circles) in seven individual rats during Phases 1 and 2 of Experiment 1.

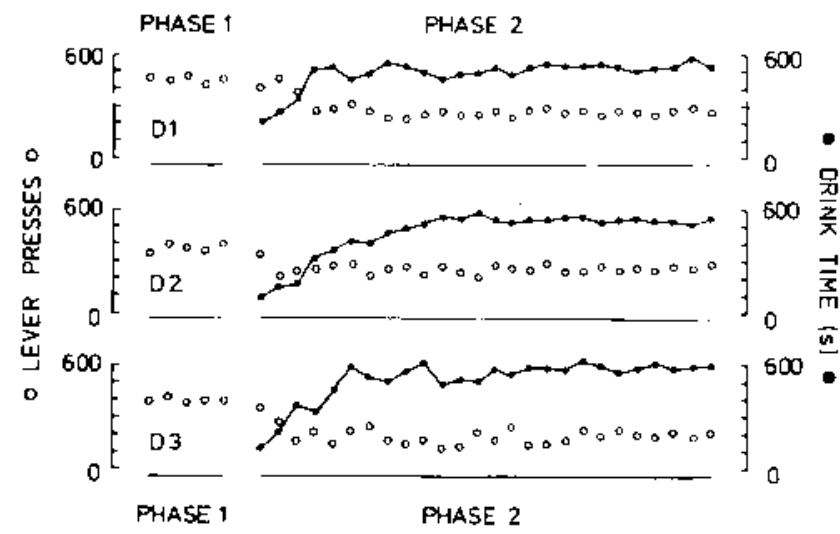

Figure 2. Number of leverpresses per session (open circles) and drinking the per seuston (filled clrcles) in three individual rats during Phases 1 and 2 of Experiment 1.

the two figures, it can be seen that in comparison with induced drinking, induced wood-chewing was slow and erratic in its initial development and, even at asymptote, was subject to large individual and day-to-day fluctuation. 


\section{Phase 3: 20\% Omission}

Figure 3 shows the effect of pellet omission on the percentage of interreinforcement intervals containing schedule-induced behavior (upper panels) and on the bout duration of schedule-induced behavior (lower panels). The left-hand panels refer to Group $C$ (wood-chewing) and the right-hand panels to Group D (drinking). Results are shown separately for successive quarters of the 60-min session, designated Q1 to Q4. $R^{+}$refers to interreinforcement intervals beginning with food delivery, while $\mathrm{R}^{-}$refers to intervals beginning with food omission. Data were pooled over the last five sessions of Phase 3 for each rat, and means and standard deviations were then taken across rats.

Considering first the upper left-hand panel of Figure 3, the results show a marked within-session decline in the percentage of interreinforcement intervals containing induced wood-chewing $[F(3,18)$ $=50.9, \mathrm{p}<.001 \mathrm{~J}$. In addition, chewing was slightly suppressed in $R^{-}$intervals relative to $R^{+}$intervals $[F(1,6)=20.9, p<.01]$. In contrast, the upper righthand panel shows no within-session change in the percentage of intervals containing schedule-induced drinking, while there was a large reduction in drinking in $\mathbf{R}^{-}$intervals in comparison with $\mathbf{R}^{*}$ intervals $[F(1,2)=278, p<.001]$.

The lower panels of Figure 3 show the "bout duration" of induced behavior: that is, the time spent chewing or drinking per $60-\mathrm{sec}$ interval, averaged only over those intervals in which chewing or drinking did occur. The lower left-hand panel shows a slight within-session decline in bout duration of induced wood-chewing $[F(3,18)=5.51, p<.05]$, but no significant reduction in chewing as a consequence of food omission. By contrast, the lower right-hand
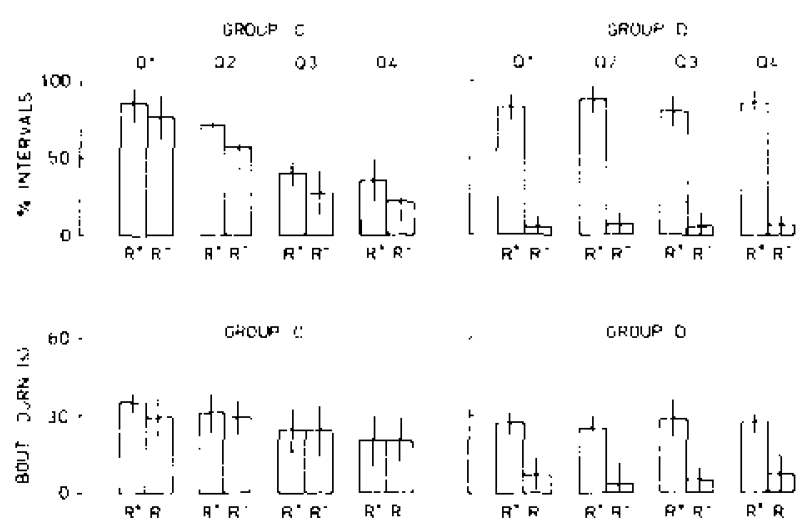

Figure 3. Effect of pellet omisation on the frequency (upper pancls) and bout duration (lower paneb) of schedule-fnduced wood-chewing (left-hand panels) and schedule-induced drinking (right-hand panels) in Phase 3 of Experiment 1. Q1 to Q4 designate successive quarters of a 60 -min session. $\mathbf{R}$ designates interreinforcement Intervals following pellet delivery; $\mathbf{R}$ deignates intervals followiog pellet omiscion (mean and SD for seven rats).

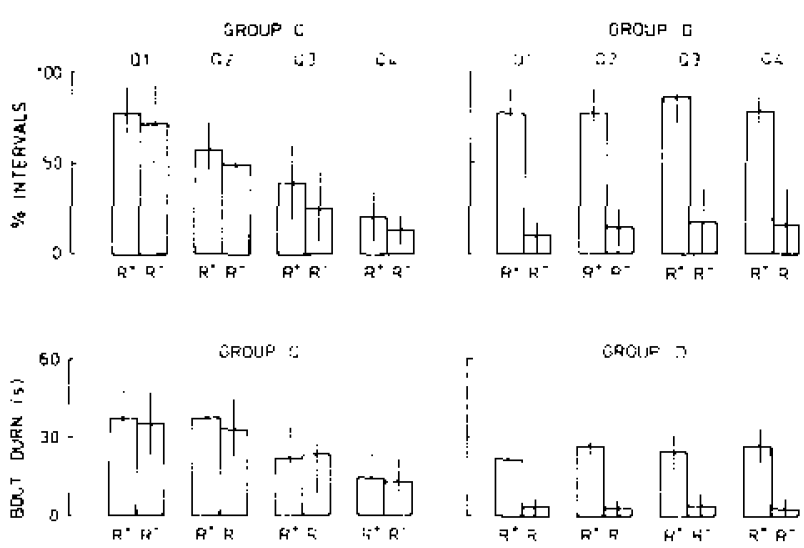

Flgure 4. Effect of pellet omiasion on the frequency and bout duration of schedale-iaduced wood-chewing and drinking in Phase 4 or Experiment 1 (conventions as in Figure 3).

panel shows no significant within-session change in bout duration of drinking, but a dramatic reduction in bout duration of drinking as a consequence of food omission $[F(1,2)=157, p<.001]$.

\section{Phase 4: 50\% Omission}

Figure 4 shows the corresponding results for Phase 4 of the experiment. In Group $C$ (left-hand panels), there was a within-session decline both in the percentage of intervals containing wood-chewing and in the bout duration of chewing $[F s(3,18)=$ 32.5 and 19.5 , ps $<.001$ ]. Omission of food caused a slight decline in the percentage of intervals containing wood-chewing $[\mathrm{F}(1,6)=6.08, \mathrm{p}<.05]$, but no significant change in the bout duration of chewing.

In Group D (right-hand panels), there was no significant within-session change either in the percentage of intervals containing induced drinking or in the bout duration of drinking. However, omission of food caused a marked decline in both measures of drinking $[\mathrm{Fs}(1,2)=113$ and 354 , ps $<.01]$.

\section{Other Observations}

When wood-chewing did occur, the behavior was surprisingly vigorous, so much so that it became necessary to replace each wood block every few sessions. Typically, the rat would grasp the wood block with one or both paws and gnaw at the edges of the block, allowing fragments of wood to drop through the floor grid (see Figure 5). Chewing sometimes continued beyond the time when food reinforcement was available, but all the rats reliably obtained at least 55 of the 60 pellets available per session.

\section{EXPERIMENT 2}

One of the best known properties of SIP is that the amount of water drunk per pellet is related to 


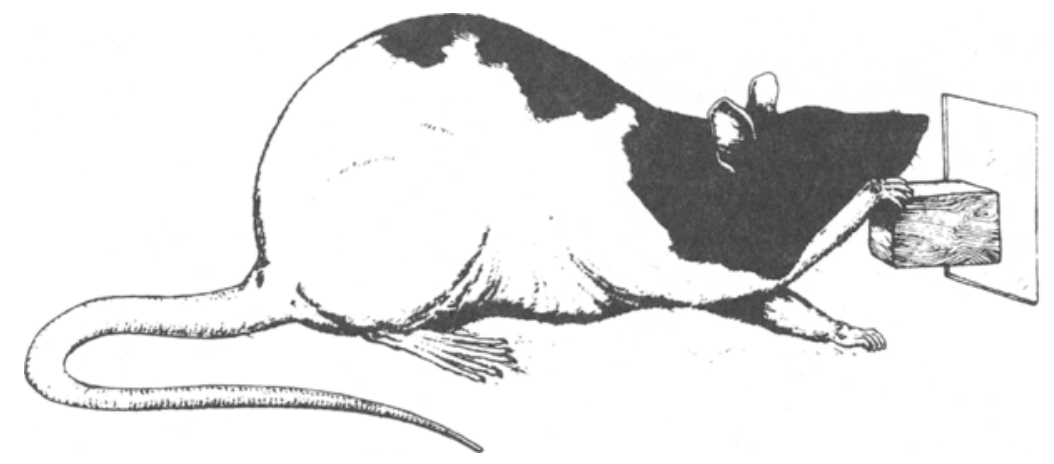

Figure 5. Typical posture adopted by rat whlle chewlog the wood block (drawn trom a vldeo recording).

reinforcement rate by an inverted U-shaped function, with maximum drinking corresponding to rates of about 25 to 100 reinforcements/h (see reviews by Allen \& Kenshalo, 1976; Roper, 1980a; Wetherington, 1979). An absolute measure of drinking (amount drunk per pellet or per session) has tended to be adopted in studies of SIP because the most striking feature of SIP is the sheer amount of water that a rat can be induced to drink (cf. Falk, 1966).

If, on the other hand, drinking is measured in terms of the amount of water ingested per unit time, a different curve results, concerning the form of which there is disagreement. Some investigators have claimed that rate of drinking is an increasing monotonic function of reinforcement rate (e.g., Cohen, 1975; Wetherington, 1979), while others have obtained a bitonic function (e.g., Allen \& Kenshalo, 1976; Heyman \& Bouzas, 1980; Roper, 1980a). Fortunately, this discrepancy seems to have resulted from nothing more than a difference in the range of reinforcement rates employed in different studies: a downturn in the curve relating drinking rate to reinforcement rate is apparent only when reinforcement rate exceeds about 300 pellets $/ \mathrm{h}$, which is larger than the highest rates employed by Cohen (1975) and by Wetherington (1979). We conclude that the rate curve for induced drinking is bitonic, with a maximum at relatively high reinforcement rates (a conclusion reached also by Heyman \& Bouzas, 1980 ).

In addition to these studies of SIP there have been several investigations of the relationship between rate of reinforcement and rate of schedule-induced aggression in pigeons (see reviews by Staddon, 1977; Wetherington, 1979). These studies also reveal a bitonic function, but with maximum rate of aggression occurring at relatively low reinforcement rates (about 20 to 60 reinforcements $/ h$ ). Finally, there is some evidence of a bitonic relationship between reinforcement rate and rate of schedule-induced escape (Brown \& Flory, 1972; Lydersen, Perkins, Thome, \& Lowman, 1980), with maximum rate of induced escape occurring at even lower reinforcement rates (about 15 reinforcements/h or less).

In Experiment 2, we examined the occurrence of induced wood-chewing under a range of reinforcement rates (FI schedules). To facilitate comparison with other studies, we present both an absolute measure of wood-chewing (total time spent chewing per session) and a rate measure (relative time spent chewing).

\section{Method}

\section{Subjects and Apparatus}

The subjects were nine naive female hooded rats (mean wejght $242 \mathrm{~g})$, housed and tested as for Group $\mathrm{C}$ in Experiment 1.

\section{Procedure}

Phase 1: Pretraining. After magazine training and shaping, the rats experienced 1060 -min sessions of FI $60-\mathrm{sec}$ food reinforcement with no wood block present, followed by 25 sessions with a wood block concurrently available. Four of the nine rats were rejected because they showed little or no wood-chewing behavior.

Phnse 2: Experimenta] testing. The remaining five rats were subjected to the following schedules: FI 60-sec, FI 5-sec, FI 30-sec, FI 60-sec, FI 120-sec, FI 180-sec, and FI 60-sec. For three of the rats, the schedules were presented in that order, and for the other two rats, they were presented in the teverse order. Ten sessions were conducted at each schedule value. All sessions jasted for 90 min and contained 30 pellet deliveries. After delivery of the 30th pellet, the lever was withdrawn, leaving the rat in a timeout condition for the remainder of the session.

\section{Results}

Figure 6a shows the total time spent wood-chewing per session, averaged over the last three sessions at each FI value for each of the five rats. The disconnected points refer to FI $60-\mathrm{sec}$ conditions that preceded and followed the main series of schedules. Rats 3, 4, and 6 experienced the schedules in ascending order; Rats 8 and 9 experienced them in descending order. In four of the rats, there was a clear bitonic relationship between time spent chewing and 
FI value, with maximum chewing either at FI $60-\mathrm{sec}$ (Rats 3, 4, and 6) or at FI $120 \mathrm{sec}$ (Rat 9). In the fifth rat (Rat 8), there was a less orderly relationship between chewing time and FI value, but there was some suggestion of a peak in chewing at FI 60 .

In Figures $6 \mathrm{~b}$ and $6 \mathrm{c}$, the total time spent chewing has been broken down to show time spent chewing during the first part of the session, when food was available (Figure 6b), and time spent chewing during the second part of the session, when the lever was withdrawn (time-out: Figure $6 \mathrm{c}$ ). In overall shape, the curves showing chewing during the FI part of the session resemble those showing total chewing: the main difference is due to absence of betweenfood chewing in the FI 5-sec condition. Chewing during time-out tended to decline as schedule value increased. This was to be expected, since the same number of reinforcements were available in all conditions, so that duration of time-out decreased as FI value increased. In general, however, relatively

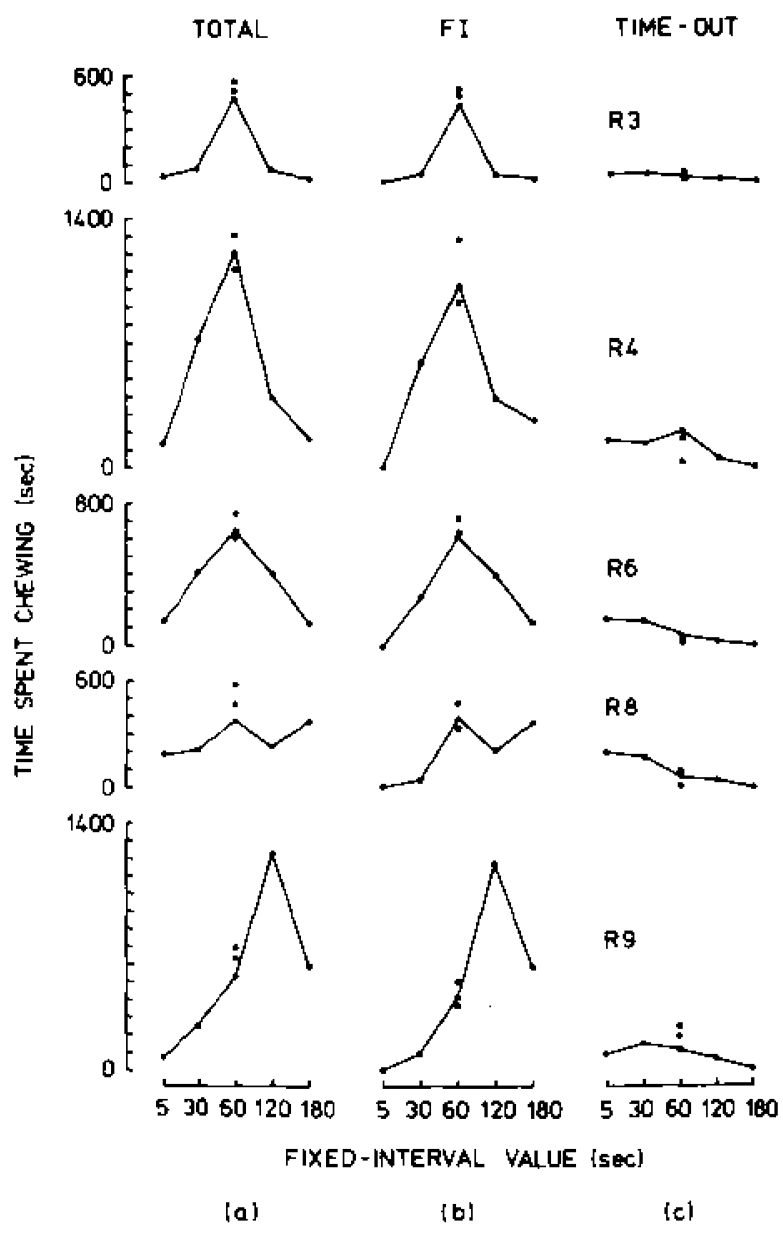

Figure 6. (a) Time spent wood-chewing per session in the individual rats in Experiment 2, th different fixed-interval values. (b) Thme spent chewing durlos the fnitial part of the wesion when food was scheduled. (c) Time spent chewing duritg the later part of the session when no food was schednled (time-out).

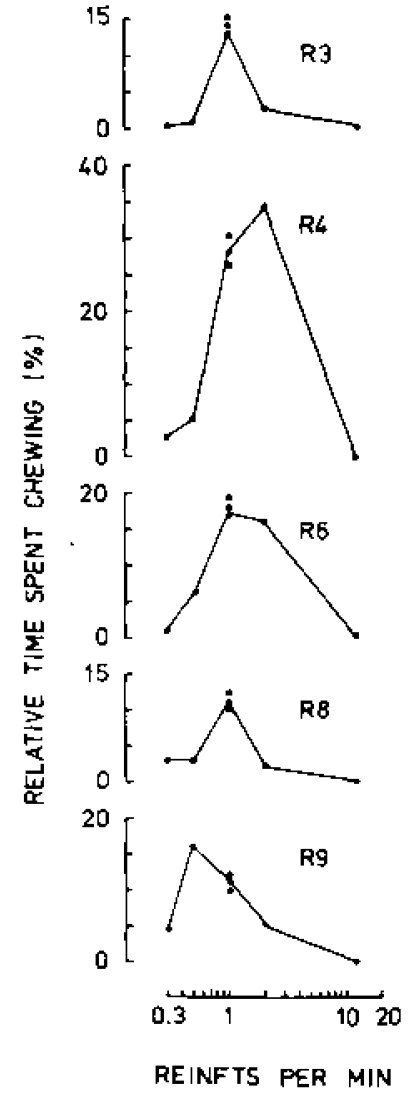

Fiqure 7. Relative time spent ehewing as a function of reinforcement rate in five individual nats in Experiment 2.

little wood-chewing occurred during the time-out part of the session.

Figure 7 shows a rate measure of chewing: namely, relative time spent chewing during the FI part of the session. This was calculated by dividing time spent chewing during the FI part of the session (as shown in Figure 6b) by time taken to obtain the $\mathbf{3 0}$ available pellets. The result was expressed as a percentage and plotted against reinforcement rate (note that the scale of the abscissa is logarithmic). In all five rats, relative time spent chewing varied bitonically with reinforcement rate, reaching a maximum at reinforcement rates of from .5 to 2.0 pellets/min (30 to 120 pellets $/ \mathrm{h}$ ).

\section{EXPERIMENT 3}

Falk's original demonstration of SIP used a response-dependent (VI) schedule, but many subsequent studies have shown that SIP develops equally readily under response-independent (e.g., FT and VT) schedules (see review by Falk, 1971). The essential condition for the appearance of induced drinking is therefore the spaced delivery of food pellets (Staddon, 1977).

Schedule-induced wood-chewing, on the other 
hand, has so far been demonstrated only in conjunction with response-dependent schedules. Furthermore, we have failed on one occasion to obtain induced chewing using an FT 60-sec schedule of food delivery (Roper \& Crossland, unpublished result, 1980), suggesting that induced chewing may require a leverpress response for its acquisition. As a step towards elucidating this anomaly, Experiment 3 investigated the necessity of a leverpress requirement for the maintenance of induced wood-chewing.

\section{Method}

\section{Subjects and Apparatus}

The subjects were five female hooded rats used in a previous study of schedule-induced wood-chewing (Roper \& Crossland, 1982). Housing and testing conditions were as in Experjments 1 and 2.

\section{Procedure}

Testing consisted of 15 60-min sessions of FI $60-\mathrm{sec}$ food reinforcement followed by 15 sessions of an FT 60-sec schedule, with concurrent free access to a wood chewometer. A response lever was available throughout the experiment, but during the FT condition leverpressing had no scheduled consequences. Because of the rats' previous experience, there was no need for pretraining.

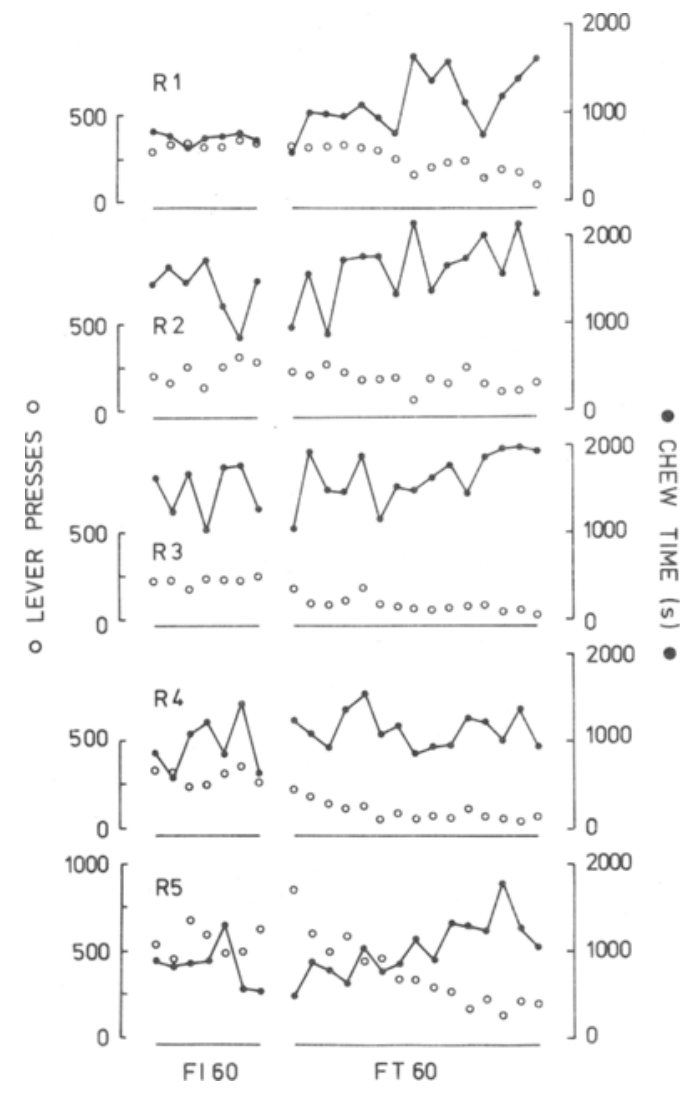

Figure \$. Number of leverpresses per seston (open circles) and time spent wood-chewing per sesslon (filled circles) in flve individual rats in Experiment 3, uader FI 60-sec and FT 60-sec schedules.

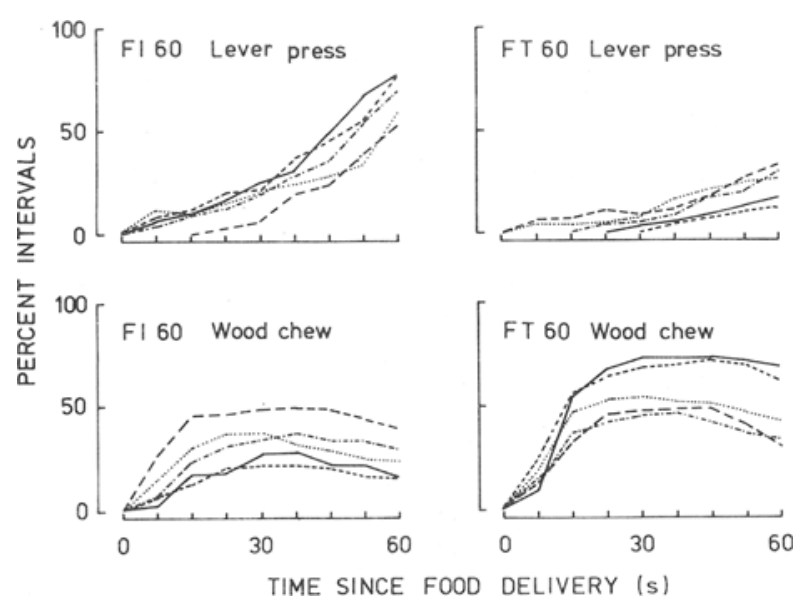

Figure 9. Temporal distribution of leverpressing and of woodchewing within the interreibforcement interval in five individusl rats, under F1 60-sec and FT 60-sec schedules.

\section{Results}

Figure 8 shows number of leverpresses per session and total chew time per session in each of the five rats, for the final sessions of FI 60 and for all sessions of FT 60 . During the FT condition, leverpressing declined in all rats, although in none of them did it cease altogether. Chew time either increased slightly during the FT condition (Rats $1,2,3$, and 5) or remained at about the same level (Rat 4).

Figure 9 shows the percentage of interfood intervals in which leverpressing or wood-chewing occurred, during successive eighths of the 60-sec interval. Data were averaged over the last three sessions of each schedule condition, in each rat. In the FI 60-sec condition (left-hand panels), leverpressing increased towards the end of the interfood interval, while wood-chewing peaked near the middle of the interval. In the FT $60-\mathrm{sec}$ condition, leverpressing was reduced and wood-chewing enhanced, but the overall shape of the distributions was not much affected. In both conditions, bouts of wood-chewing were often still in progress when the next food pellet became available.

\section{DISCUSSION}

Taken together with previous studies of induced chewing (Killeen, 1975; Roper, 1978; Roper \& Crossland, 1982), our results suggest that induced chewing differs from SIP in some respects but resembles it in others. The question to be asked (and to which we have as yet no definite answer) is whether these apparent similarities and differences tell us anything important about schedule induction, or whether they merely reflect relatively trivial characteristics of the activities in question or of the procedures employed. 
Experiment 1 suggests that induced wood-chewing is in various respects a less reliable phenomenon than induced drinking (see also Roper, 1978; Roper \& Crossland, 1982). Specifically, induced chewing is slow to develop and fails to appear at all in a substantial proportion of subjects; at asymptote, it displays considerable between-subjects and day-to-day variability; it declines markedly within the session; and even at its strongest it occurs in a relatively small proportion of interfood intervals.

Two points seem worth making about these results. First, the differences between induced chewing and induced drinking are ones of degree rather than of kind. An apparent exception to this is that in Experiment 1 chewing declined markedly within the session, whereas drinking did not; but some investigators have reported within-session satiation of induced drinking (e.g., Hymowitz \& Freed, 1972; Staddon \& Ayres, 1975). Second, the betweensubjects variability displayed by induced chewing, both during acquisition and at asymptote, is more reminiscent of induced aggression in pigeons than of induced drinking in rats. For example, Looney and Cohen (1978) found that induced aggression could take up to 30 sessions to reach asymptote, and that it failed to occur in almost a third of subjects. It would be interesting to know whether induced aggression also shows a within-session decline, but as far as we know no one has yet commented on this aspect of the behavior.

Induced aggression is responsive to refinements of procedure (e.g., Looney \& Cohen, 1978; Looney, Cohen, \& Yoburn, 1976), and the same may prove to be true of induced chewing. However, it is possible that the differences between chewing, aggression, and drinking are not just procedural artifacts, and next we discuss an alternative interpretation in the context of the effects of food omission.

Much of the theoretical speculation about SIP has concerned the precise role played by delivery of a food pellet. Is drinking induced by the various stimuli and/or responses that accompany food ingestion (e.g., taste and texture of food, chewing, and swallowing), or is drinking induced because pellet delivery constitutes a discriminative stimulus signaling low probability of reinforcement, or both?

If drinking follows eating because eating signals a period of low reinforcer probability (e.g., Staddon, 1977), then drinking should follow any other stimulus that has the same signal value. This prediction has been tested using second-order schedules in which one component terminates in food while another terminates in a nonfood discriminative stimulus. The relevant studies have yielded diverse results, but all agree that drinking is considerably reduced, but not absolutely abolished, by omission of food (see review by Corfield-Sumner, Blackman, \& Stainer, 1977). In Experiment 1, we confirmed that omission of food substantially reduces both the probability and the bout duration of drinking, but found that omission had relatively little effect on the probability of induced wood-chewing and no significant effect on its bout duration.

The fact that induced drinking is reduced, but not eliminated, by omission of food suggests that both the eliciting and the discriminative properties of food presentation contribute to the maintenance of SIP (see also Alferink, Bartness, \& Harder, 1980, and Heyman \& Bouzas, 1980, for a similar conclusion). One approach to explaining the differences between induced chewing, drinking, and aggression might therefore be to postulate differences in the relative contribution of eliciting and discriminative factors. There is good reason to presuppose a strong tendency for eating to elicit drinking, because bouts of drinking often follow bouts of eating when food and water are freely avajlable to a bungry rat (e.g., Fitzsimons \& LeMagnen, 1969; Oatley, 1971; Roper, 1980b). Wood-chewing, on the other hand, has virtually zero probability when food is freely available, suggesting that eating is only a weak elicitor of chewing. If we suppose that strength of induced behavior is some function both of the eliciting value of food and of its discriminative value, and that its eliciting value is lower for chewing than for drinking, this might explain both why chewing is less strongly induced by food schedules to begin with and why chewing is relatively less distupted by the substitution of a nonfood discriminative stimulus (which presumably has an eliciting value of zero). According to this formulation, the differences between induced chewing and induced drinking result from intrinsic differences in the activities themselves (or more accurately, in their relationship to eating), rather than from procedural side effects. However, it is unlikely that this can completely account for the observed differences, because following food omission chewing and drinking were not reduced to the same absolute probability of occurrence (see Figures 3 and 4).

Experiment 2 showed that amount of induced chewing per session and rate of chewing per unit time both vary in a bitonic manner as a function of reinforcement rate. Previous studies have revealed bitonic functions both for amount of induced drinking per session and for rate of induced drinking and of induced aggression per unit time (for recent reviews, see Heyman \& Bouzas, 1980, Roper, 1980a, and Wetherington, 1979). (It has sometimes been claimed that rate of induced drinking is related monotonically to rate of reinforcement, but this is because the relevant studies have not used sufficiently high rates of reinforcement to reveal the descending limb of the bitonic curve; see Heyman \& Bouzas, 1980.)

At the same time, the reinforcement rate found 
to support maximum induced behavior differs markedly between induced drinking, on the one hand, and jnduced chewing and aggression, on the other. Rate of induced drinking peaks at about 250 to 350 reinforcements/h (Heyman \& Bouzas, 1980; Roper, 1980a), while rate of induced chewing peaks at 30 to 120 reinforcements/h (see Figure 7 ) and rate of induced aggression peaks at 20 to 60 reinforcements $/ \mathrm{h}$ (Staddon, 1977, Figure 5).

These quantitative discrepancies may well result from procedural differences. For example, in seeking to explain differences between rate of drinking in rats and rate of aggression in pigeons, Heyman and Bouzas note that the relevant pigeon studies involved a changeover delay such that the occurrence of attack within the last $15 \mathrm{sec}$ of an interfood interval delayed food by a further $15 \mathrm{sec}$. Wetherington (1979), on the other hand, offers a more theoretical explanation. She suggests that the apparent differences may diminish if rate of induced behavior is computed on the basis of time available for induced behavior rather than on the basis of total session duration. At the very least, this means subtracting from session duration the time spent eating, but Wetherington also suggests taking into account the different latencies of different induced behaviors. For example, chewing shows a more gradual rise in probability within the interfood interval than does drinking (Roper, 1978; Roper \& Crossland, 1982), so that a smaller proportion of the interfood interval is available for chewing. Similarly, different induced behaviors may overlap to different extents with operant or other terminal responses occurring at the end of the interfood interval. It remains to be seen whether this approach is useful. An obvious problem is that the argument could easily become circular: Is the time available for induced behavior constrained by other activities, or vice versa?

Experiment 3 showed that induced chewing can be maintained by a response-independent (FT) schedule, but leaves open the question of whether a similar schedule will support the acquisition of chewing. We have failed on one occasion to obtain induced chewing in naive rats using an FT 60-sec schedule (Roper \& Crossland, unpublished), but this single negative result can hardly be taken as conclusive. It may be worth noting that, while induced aggression in pigeons has occasionally been obtained using FT schedules (Dove, 1976; Flory, 1969; Looney \& Cohen, 1978), the vast majority of studies of this behavior have used response-dependent schedules. Thus, although the presence of an operant requirement is irrelevant to the acquisition of induced drinking (e.g., Falk, 1971), this may not be the case for other induced behaviors.

Studies of different induced behaviors inevitably differ in matters of procedural detail, so that inter- pretation of the results will always be controversial. However, this does not necessarily mean that attempts to compare the properties of different induced activities are doomed to failure. The present study shows that induced wood-chewing has important features in common with induced drinking and aggression, and this provides powerful support for the idea that schedule induction is a general phenomenon. With care, it may also be possible to account satisfactorily for the quantitative differences.

\section{REFERENCES}

Alferink, L. A., Bartwe b8, T. J., \& Hanber, S. R. Control of the temporal location of polydipsic licking in the rat. Journal of the Experimental A nalysis of Behavior, 1980, 33, 119-129.

ALLe N, J. D., Kenghalo, D. R., JR. Schedule-induced drinking as a function of interreinforcement interval in the rhesus monkey. Journal of the Experimental Analysis of Behavior, $1976,26,257-267$.

Allew, J. D., Porter, J. H., \& Anazie, R. Schedule-induced drinking as a function of percentage reinforcement. Journal of the Experimental A nalysis of Behovior, 1975, 23, 223-232.

Brown, T. G., Flony, $R$. K. Schedule-induced eacape from fixed-interval reinforcement. Journal of the Experimental Analysis of Behavior, 1972, 17, 395-403.

CoHev, 1. L. The reinforcement value of schedule-induced drinking. Journal of the Experimental Amalysis of Behavior, 1975, $23,37-44$.

Corfieldo-Sumner, P. K., Blackman, D. E., \& Stainen, G. Polydipsia induced in rats by second-order schedules of reinforcement. Journal of the Experimental Analysis of Behavior, $1977,27,265-273$.

Dove, L. D. Relation between level of food deprivation and rate of schedule-induced attack. Jotrnal of the Experimental Analysis of Behavior, 1976, 29, 63-68.

FatK, J. L. Production of polydipsia in normal rats by an in. termittent food schedule. Science, 1961, 133, 195-196.

FaLk, J. L. Schedule-induced polydipsin as a function of fixed interval length. Journal of the Experimental Analysis of Behavior, 1966, 9, 37-39.

FALK. J. L. The nature and determinants of adjunctive behavior. Physiology \& Behavior, 1971, 6, 577.588.

FAtK, J. L. The origin and functions of adjunctive behavior. Animal Learning \& Behavior, 1977, 5, 325-335.

Fitzsimons, T. J., \& LeMAONEN, J. Eating as a regulatory control of drinking in the rat. Journal of Comparative and Physiological Psychology, 1969, 67, 273-283.

FLony, R. Attack behavior as a function of minimum inter-food interval. Journal of the Experimental Analysis of Behavior, $1969,12,825-828$.

Hryman, G. M., Bouzas. A. Context dependent changes in the reinforcement value of schedule-induced drinking. Journal of the Experimental Analysis of Behavior, 1980, 33, 327-335.

Hrmowitz, N., \& FaEED, E. X. Inconstancy of drinking butsts during schedule-induced polydipsia. Psychonomic Science, $1972,28,283-284$.

KILle EN, P. On the temporal control of behavior. Psychological Revlew, 1979, 82, 89-115.

LoONey, T, A., \& Cohen, L. D. Schedule-induced attack as a function of length of exposure to fixed-time $90-\mathrm{sec}$ schedule. Bulletin of the Psychonomic Society, 1978, 12, 320-322.

Looner, T, A., Cohen, P. S., \& Yoburn, B. C. Variables affecting establishment of schedule-induced attack on pictorial targets in White King pigeons. Joumal of the Experimental Analysis of Behavior, 1976, 26, 349-360.

Lyderaen, T., Penking, D., Thome, S., Lowuan, E. Choice 
of timeout during response-independent food schedules. Journal of the Experimental Analysis of Behavior, 1980, 33, 59.76.

OATLEY, K. Dissociation of the circadian drinking pattern from eating. Nature, 1971, 229, 494-496.

Roper, T. J. Diversity and substitutability of adjunctive activities under fixed-interval schedules of food reinforcement. Journal of the Experimental Analysis of Behavior, 1978, 30, 83-96.

ROPER. T. J. Changes in rate of schedule-induced behavior in rats as a function of fixed-interval schedule. Quarierly Journal of Experimentol Psychology, 1980, 32, 159-170. (a)

ROPER, T. J. Behavior of rats during self-initiated pauses in feeding and drinking, and during periodic response-independent delivery of food and water. Quarterly Jowrnal of Experimental Psychology, 1980, 32, 459.472. (b)

Roper, T. J. What is meant by the term "schedule-induced" and how general is schedule induction? Animal Learning a Behavior, 1981, 9, 433-440.
Roper, T. J., \& Crossiand, G. Schedule-induced wood-chewing in rats and its dependence on body weight. Animal Learning \& Behavior, 1982, 10, 65.71.

ROPER, T. J., \& Nieto, J. Schedule-induced drinking and other behavior in the rat, as a function of body-weight deficit. Physiology \& Behavior, 1979, 23, 673-678.

Staddon, J. E. R. Schedule-induced behavior. In W. K. Honig 2 J. E. R. Staddon (Eds.), Handbook of operant behavior. Englewood Cliffs, N.J: Prentice-Hall, 1977.

Staddon, J. E. R., AYnes, S. L. Sequential and temporal properties of behavior induced by a schedule of periodic food delivery. Behaviour, 1975, 54, 26-49.

Wetherington, C. L. Schedule-induced drinking: Rate of food delivery and Herrnstein's equation. Journal of the Experimental Analysis of Behavior, 1979, 32, 323-333.

(Manuscript teceived March 26, 1982; revision accepted for publication August 30, 1982.) 